\title{
A NOTE ON INTEGERS OF THE FORM $2^{n}+c p$
}

\author{
ZHI-WEI SUN ${ }^{1}$ AND SI-MAN YANG ${ }^{2}$ \\ ${ }^{1}$ Department of Mathematics, Nanjing University, Nanjing 210093, \\ The People's Republic of China (zwsun@nju.edu.cn) \\ ${ }^{2}$ Department of Mathematics, National University of Singapore, \\ 2 Science Drive 2, Singapore 117543
}

(Received 15 February 2000)

\begin{abstract}
In 1950 Erdös proved that if $x \equiv 2036812(\bmod 5592405)$ and $x \equiv 3(\bmod 62)$, then $x$ is not of the form $2^{n}+p$, where $n$ is a non-negative integer and $p$ is a prime. In this note we present a theorem on integers of the form $2^{n}+c p$, in particular we completely determine all those integers $c$ relatively prime to 5592405 such that the residue class $2036812(\bmod 5592405)$ contains integers of the form $2^{n}+c p$.
\end{abstract}

Keywords: integers of the form $2^{n}+c p$; cover of $\mathbb{Z}$; residue class; primitive prime divisor

AMS 2000 Mathematics subject classification: Primary 11P32

Secondary 11A07; 11B25; 11B75

In 1849 de Polignac [4] claimed that any sufficiently large odd integer is of the form $2^{n}+p$, where $n$ is a non-negative integer and $p$ is a prime. Erdös [5] proved that any integer congruent to $2036812 \bmod 5592405$ and $3 \bmod 62$ cannot be the sum of a power of two and a prime, a clear proof of this result was presented by Sierpiński $[\mathbf{1 1}]($ see $[\mathbf{3}, \mathbf{7}, \mathbf{8}, \mathbf{1 3}]$ for further developments). In his ingenious proof, Erdös introduced the concept of cover of $\mathbb{Z}$. For $a, n \in \mathbb{Z}$ with $n>0$ we put

$$
a(\bmod n)=\{x \in \mathbb{Z}: x \equiv a(\bmod n)\}
$$

and call it a residue class (with modulus $n$ ). A finite system

$$
A=\left\{a_{s}\left(\bmod n_{s}\right)\right\}_{s=1}^{k}
$$

of such classes is said to be a cover (of $\mathbb{Z})$ if $\bigcup_{s=1}^{k} a_{s}\left(\bmod n_{s}\right)=\mathbb{Z}$. If (1) forms a cover but none of its proper subsystems does, then we say that (1) is a minimal cover. For problems and results concerning covers of $\mathbb{Z}$ see $[\mathbf{6}],[\mathbf{9}]$ and the introduction of [12].

A well-known result of Bang [1] (also rediscovered by Zsigmondy [15] and Birkhoff and Vandiver [2]) states that for each integer $n>1$ with $n \neq 6$, there exists a prime factor of $2^{n}-1$ not dividing $2^{m}-1$ for any $0<m<n$, such a prime is called a primitive (prime) divisor of $2^{n}-1$. In [10] the reader can find all prime divisors of $2^{n}-1$ with $n \leqslant 22$.

Our main result in this note is the following theorem. 
Theorem 1. Let (1) be a minimal cover with $0 \leqslant a_{s}<n_{s}$ for $s=1, \ldots, k$. Suppose that distinct primes $p_{1}, \ldots, p_{k}$ are primitive divisors of $2^{n_{1}}-1, \ldots, 2^{n_{k}}-1$, respectively. Put $\bigcap_{s=1}^{k} 2^{a_{s}}\left(\bmod p_{s}\right)=a(\bmod d)$, where $a \in \mathbb{Z}$ and $d=p_{1} \cdots p_{k}$, and write

$$
\left(a_{t}\left(\bmod n_{t}\right) \backslash \bigcup_{\substack{s=1 \\ s \neq t}}^{k} a_{s}\left(\bmod n_{s}\right)\right) \cap\{0,1, \ldots, N-1\}=\left\{b_{1}^{(t)}, \ldots, b_{l_{t}}^{(t)}\right\}
$$

for $t=1, \ldots, k$, where $N$ is the least common multiple $\left[n_{1}, \ldots, n_{k}\right]$ of the moduli $n_{1}, \ldots, n_{k}$. Set

$$
S(A)=\bigcup_{t=1}^{k} \bigcup_{j=1}^{l_{t}} \frac{a-2^{b_{j}^{(t)}}}{p_{t}}\left(\bmod \frac{d}{p_{t}}\right)
$$

where all the $\left(a-2^{b_{j}^{(t)}}\right) / p_{t}$ are integers. Then an integer $c$ divisible by none of $p_{1}, \ldots, p_{k}$ belongs to $S(A)$ if and only if $a(\bmod d)$ contains integers of the form $2^{n}+c p$, where $n \geqslant 0$ is an integer and $p$ is a prime.

Proof. Let $1 \leqslant t \leqslant k$ and $1 \leqslant j \leqslant l_{t}$. As $b_{j}^{(t)} \equiv a_{t}\left(\bmod n_{t}\right), a \equiv 2^{a_{t}} \equiv 2^{b_{j}^{(t)}}\left(\bmod p_{t}\right)$. Let $c \equiv\left(a-2^{b_{j}^{(t)}}\right) / p_{t}\left(\bmod d / p_{t}\right)$. Since $d=p_{1} \cdots p_{k}$ divides $2^{N}-1$, for any non-negative integer $n \equiv b_{j}^{(t)}(\bmod N)$ we have

$$
2^{n}+c p_{t} \equiv 2^{b_{j}^{(t)}}+c p_{t} \equiv a(\bmod d) .
$$

Next we prove the sufficiency. Let $c$ be an integer relatively prime to $d$. Suppose that $2^{n}+c p \equiv a(\bmod d)$ for some integer $n \geqslant 0$ and prime $p$. Since $(1)$ forms a cover, $n \equiv a_{t}\left(\bmod n_{t}\right)$ for some $1 \leqslant t \leqslant k$. Observe that $2^{n} \equiv 2^{a_{t}} \equiv a\left(\bmod p_{t}\right)$. So $p_{t} \mid c p$, and hence $p=p_{t}$. For any $s=1, \ldots, k$ with $s \neq t$, we have $p \neq p_{s}$ and thus $n \not \equiv a_{s}\left(\bmod n_{s}\right)$. Therefore $n \equiv b_{j}^{(t)}(\bmod N)$ for some $j=1, \ldots, l_{t}$. It follows that

$$
c p_{t}=c p \equiv a-2^{n} \equiv a-2^{b_{j}^{(t)}}(\bmod d),
$$

i.e.

$$
c \equiv \frac{a-2^{b_{j}^{(t)}}}{p_{t}}\left(\bmod \frac{d}{p_{t}}\right)
$$

So $c \in S(A)$.

The proof is now complete.

Remark 2. Note that $\left(a-2^{b_{j}^{(t)}}\right) / p_{t}$ is relatively prime to $d / p_{t}$, for, if $1 \leqslant s \leqslant k$ and $s \neq t$, then $b_{j}^{(t)} \not \equiv a_{s}\left(\bmod n_{s}\right)$, and hence $a-2^{b_{j}^{(t)}} \not \equiv a-2^{a_{s}} \equiv 0\left(\bmod p_{s}\right)$. In practice we can split $\left(a-2^{b_{j}^{(t)}}\right) / p_{t}\left(\bmod d / p_{t}\right)$ into $p_{t}$ residue classes modulo $d$, exactly one of which contains only multiples of $p_{t}$ and should be deleted for our purpose.

Remark 3. Under the conditions of Theorem 1, the authors [14] showed that if $c$ is divisible by a unique prime among $p_{1}, \ldots, p_{k}$, then there exists a positive integer $n$ such that $2^{n}+c p \in a(\bmod d)$ for infinitely many primes $p$. 
Erdös used the following cover

$$
B=\{0(\bmod 2), 0(\bmod 3), 1(\bmod 4), 3(\bmod 8), 7(\bmod 12), 23(\bmod 24)\}
$$

to get counterexamples to the claim of de Polignac. It is easy to check that $2^{2}-1,2^{3}-1$, $2^{4}-1,2^{8}-1,2^{12}-1,2^{24}-1$ have primitive prime divisors

$$
3, \quad 7, \quad 5, \quad 17, \quad 13, \quad 241
$$

respectively. Notice that the intersection

$$
2^{0}(\bmod 3) \cap 2^{0}(\bmod 7) \cap 2(\bmod 5) \cap 2^{3}(\bmod 17) \cap 2^{7}(\bmod 13) \cap 2^{23}(\bmod 241)
$$

is $2036812(\bmod 5592405)$. Erdös showed that

$$
2036812(\bmod 5592405) \cap 1(\bmod 2) \cap 3(\bmod 31)
$$

contains no integers of the form $2^{n}+p$. Our Theorem 1 yields the following complete result.

Corollary 4. Let $c$ be an integer relatively prime to

$$
3 \times 5 \times 7 \times 13 \times 17 \times 241=5592405 \text {. }
$$

Then the residue class $2036812(\bmod 5592405)$ contains integers of the form $2^{n}+c p$, with $n$ being a non-negative integer and $p$ being a prime, if and only if $c$ is congruent to one of the following numbers modulo 5592405 :

$\begin{array}{rrrrrrr}20054 & 43259 & 66464 & 89669 & 112874 & 119692 & 136079 \\ 156668 & 159284 & 182489 & 205694 & 228899 & 252104 & 275309 \\ 286292 & 298514 & 321719 & 344924 & 368129 & 381148 & 391334 \\ 405724 & 407356 & 407362 & 414539 & 437744 & 448657 & 460949 \\ 484154 & 507359 & 530564 & 553769 & 576974 & 586853 & 600179 \\ 623384 & 646589 & 657092 & 669794 & 678596 & 678932 & 692999 \\ 716204 & 739409 & 762614 & 777622 & 785819 & 809024 & 832229 \\ 855434 & 878639 & 901844 & 925049 & 948254 & 971459 & 994664 \\ 1017038 & 1017869 & 1041074 & 1064279 & 1085207 & 1087484 & 1106587 \\ 1110689 & 1133894 & 1157099 & 1180304 & 1203509 & 1226714 & 1249919 \\ 1273124 & 1296329 & 1319534 & 1342739 & 1365944 & 1389149 & 1412354 \\ 1435552 & 1435559 & 1447223 & 1458764 & 1481969 & 1499629 & 1505174 \\ 1525837 & 1525843 & 1528379 & 1551584 & 1574789 & 1597994 & 1621199 \\ 1644404 & 1667609 & 1690814 & 1714019 & 1737224 & 1760429 & 1764517 \\ 1783634 & 1806839 & 1830044 & 1853249 & 1876454 & 1884122 & 1899659\end{array}$




$\begin{array}{lllllll}1922864 & 1946069 & 1969274 & 1992479 & 2015684 & 2038889 & 2062094 \\ 2085299 & 2108504 & 2131709 & 2154914 & 2178119 & 2193547 & 2201324 \\ 2224529 & 2247734 & 2270939 & 2294144 & 2307593 & 2317349 & 2340554 \\ 2363759 & 2386964 & 2410169 & 2422447 & 2433374 & 2456579 & 2479784 \\ 2502989 & 2526194 & 2537611 & 2542987 & 2543071 & 2549399 & 2572604 \\ 2595809 & 2619014 & 2642219 & 2642686 & 2644318 & 2644324 & 2665424 \\ 2688629 & 2711834 & 2735039 & 2737778 & 2751412 & 2758244 & 2781449 \\ 2804654 & 2827859 & 2851064 & 2874269 & 2897474 & 2943884 & 2967089 \\ 2990294 & 3009106 & 3013499 & 3036704 & 3059909 & 3080377 & 3083114 \\ 3106319 & 3129524 & 3152729 & 3167963 & 3175934 & 3199139 & 3222344 \\ 3245549 & 3268754 & 3291959 & 3315164 & 3338369 & 3361574 & 3384779 \\ 3407984 & 3409342 & 3431189 & 3454394 & 3477599 & 3481952 & 3500804 \\ 3524009 & 3547214 & 3570419 & 3593624 & 3598148 & 3616829 & 3640034 \\ 3663239 & 3686444 & 3709649 & 3732854 & 3736591 & 3738307 & 3756059 \\ 3761167 & 3762799 & 3779264 & 3802469 & 3825674 & 3848879 & 3872084 \\ 3895289 & 3918494 & 3941699 & 3964904 & 3988109 & 4011314 & 4028333 \\ 4034519 & 4057682 & 4057724 & 4067272 & 4080929 & 4104134 & 4127339 \\ 4150544 & 4173749 & 4196954 & 4220159 & 4243364 & 4266569 & 4280867 \\ 4289774 & 4312979 & 4336184 & 4359389 & 4382594 & 4385362 & 4396237 \\ 4401746 & 4405799 & 4406866 & 4407122 & 4407202 & 4407206 & 4429004 \\ 4452209 & 4458518 & 4475414 & 4498619 & 4521824 & 4545029 & 4568234 \\ 4591439 & 4614644 & 4637849 & 4661054 & 4684259 & 4707464 & 4725202 \\ 4730669 & 4753874 & 4777079 & 4800284 & 4823489 & 4846694 & 4855702 \\ 4869899 & 4873241 & 4879648 & 4881286 & 4888703 & 4893104 & 4916309 \\ 4939514 & 4962719 & 4985924 & 5009129 & 5032334 & 5054167 & 5055539 \\ 5078744 & 5079782 & 5101949 & 5125154 & 5148359 & 5171564 & 5194769 \\ 5217974 & 5241179 & 5264384 & 5287589 & 5310794 & 5318888 & 5333999 \\ 5357204 & 5380409 & 5383132 & 5403614 & 5426819 & 5450024 & 5473229 \\ 5496434 & 5519639 & 5542844 & 5566049 & 5589254 & & \end{array}$

Proof. Note that system $B$ in (4) forms a minimal cover with

$$
a_{1}=0, \quad a_{2}=0, \quad a_{3}=1, \quad a_{4}=3, \quad a_{5}=7, \quad a_{6}=23
$$

and

$$
n_{1}=2, \quad n_{2}=3, \quad n_{3}=4, \quad n_{4}=8, \quad n_{5}=12, \quad n_{6}=24 .
$$

Recall that $3,7,5,17,13,241$ are primitive prime divisors of $2^{n_{1}}-1, \ldots, 2^{n_{6}}-1$, respectively. Obviously $\left[n_{1}, \ldots, n_{6}\right]=24$. Let $R=\{0,1, \ldots, 23\}$ and

$$
S_{t}=a_{t}\left(\bmod n_{t}\right) \backslash \bigcup_{\substack{s=1 \\ s \neq t}}^{6} a_{s}\left(\bmod n_{s}\right) \quad \text { for } t=1, \ldots, 6 .
$$


Then

$$
\begin{array}{ll}
S_{1}=0(\bmod 2) \backslash 0(\bmod 3), & S_{1} \cap R=\{2,4,8,10,14,16,20,22\} ; \\
S_{2}=0(\bmod 3) \backslash(0(\bmod 2) \cup 1(\bmod 4) \cup 3(\bmod 8)), & S_{2} \cap R=\{15\} ; \\
S_{3}=1(\bmod 4) \backslash 0(\bmod 3), & S_{3} \cap R=\{1,5,13,17\} ; \\
S_{4}=3(\bmod 8) \backslash(0(\bmod 3) \cup 7(\bmod 12)), & S_{4} \cap R=\{11\} ; \\
S_{5}=7(\bmod 12) \backslash 3(\bmod 8), & S_{5} \cap R=\{7\} ; \\
S_{6}=23(\bmod 24), & S_{6} \cap R=\{23\} .
\end{array}
$$

Let $d=5592405$. By computation we find that $S(B)$ consists of the following residue classes:

$678936,678932,678852,678596,673476,657092,329412,1144971 \bmod d / 3$;

$286292(\bmod d / 7) ; \quad 407362,407356,405724,381148 \bmod d / 5 ;$

$119692(\bmod d / 17) ; \quad 156668(\bmod d / 13) ; \quad 20054(\bmod d / 241)$.

In view of Theorem 1 and Remark 2, we can now obtain the desired result through trivial calculations.

Remark 5. Observe that $5589254 \equiv-3151(\bmod 5592405)$. By Corollary 4, for any integer $c \in[-3150,20054)$ divisible by none of $3,5,7,13,17,241$, the residue class $20036812(\bmod 5592405)$ contains no integers of the form $2^{n}+c p$, where $n \geqslant 0$ is an integer and $p$ is a prime.

Acknowledgements. Z.-W.S. was supported by the Teaching and Research Award Program for Outstanding Young Teachers in Higher Education Institutions of MOE, and the National Natural Science Foundation of the People's Republic of China. The authors are indebted to the referee for his helpful suggestions.

\section{References}

1. A. S. BAng, Taltheoretiske Undersgelser, Tidsskrift Mat. 4 (1886), 70-80, 130-137.

2. G. D. Birkhoff And H. S. Vandiver, On the integral divisors of $a^{n}-b^{n}$, Ann. Math. 5 (1904), 173-180.

3. R. Crocker, On a sum of a prime and two powers of two, Pac. J. Math. 36 (1971), 103-107.

4. A. De Polignac, Recherches nouvelles sur les nombres premiers, C. R. Acad. Sci. Paris Sér. I 29 (1849), 397-401, 738-739.

5. P. ERDÖs, On integers of the form $2^{k}+p$ and some related problems, Summa Brasil. Math. 2 (1950), 113-123.

6. P. ERDÖs, Problems and results in number theory, in Recent progress in analytic number theory (ed. H. Halberstam and C. Holley), vol. 1, pp. 1-13 (Academic, 1981).

7. P. X. Gallagher, Primes and powers of 2, Invent. Math. 29 (1975), 125-142.

8. A. Granville and K. Soundararajan, A binary additive problem of Erdös and the order of $2 \bmod p^{2}$, Ramanujan J. 2 (1998), 283-298.

9. R. K. GuY, Unsolved problems in number theory, 2nd edn, pp. 251-256 (Springer, 1994). 
10. D. RICHARD, All arithmetical sets of powers of primes are first-order definable in terms of the successor function and the coprimeness predicate, Discrete Math. 53 (1985), 221-247.

11. W. SierPiński, Elementary theory of numbers, pp. 445-448 (PWN-Polish Scientific Publishers, North-Holland, Amsterdam, 1987).

12. Z.-W. Sun, Covering the integers by arithmetic sequences, II, Trans. Am. Math. Soc. 348 (1996), 4279-4320.

13. Z.-W. Sun, On integers not of the form $p^{a} \pm q^{b}$, Proc. Am. Math. Soc. 128 (2000), 997-1002.

14. S.-M. YANG AND Z.-W. Sun, Covers with less than 10 moduli and their applications, J. Southeast Univ. (English edition) 14(2) (1998), 106-114.

15. K. Zsigmondy, Zur Theorie der Potenzreste, Monatsh. Math. 3 (1892), 265-284. 